\title{
Application of Virtual Reality Head Mounted Display for Investigation of Movement: a Novel Effect of Orientation of Attention
}

\author{
Brendan Quinlivan \\ Trinity College Dublin, Ireland \\ John Butler \\ Technological University Dubln, john.s.butler@tudublin.ie \\ Ines Beiser \\ St Vincent's University Hospital
}

See next page for additional authors

Follow this and additional works at: https://arrow.tudublin.ie/scschmatart

Part of the Mathematics Commons, and the Neuroscience and Neurobiology Commons

\section{Recommended Citation}

Quinlivan, B. (2016) Application of virtual reality head mounted display for investigation of movement: a novel effect of orientation of attention, Journal of Neural Engineering 135560062016.

This Article is brought to you for free and open access by the School of Mathematics at ARROW@TU Dublin. It has been accepted for inclusion in Articles by an authorized administrator of ARROW@TU Dublin. For more information, please contact arrow.admin@tudublin.ie, aisling.coyne@tudublin.ie,gerard.connolly@tudublin.ie.

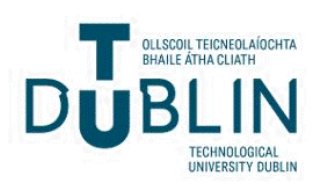




\section{Authors}

Brendan Quinlivan, John Butler, Ines Beiser, Laura Williams, Eavan McGovern, Sean O'Riordan, Michael Hutchinson, and Richard Reilly 


\title{
Application of virtual reality head mounted display for investigation of movement: a novel effect of orientation of attention
}

\author{
Brendan Quinlivan ${ }^{1,6}$, John S Butler ${ }^{2}$, Ines Beiser ${ }^{3}$, Laura Williams ${ }^{3}$, \\ Eavan McGovern ${ }^{3}$, Sean O'Riordan ${ }^{3}$, Michael Hutchinson ${ }^{4}$ and \\ Richard B Reilly ${ }^{5,6}$ \\ ${ }^{1}$ School of Engineering, Trinity College, Dublin, Ireland \\ ${ }^{2}$ School of Mathematical Sciences, Dublin Institute of Technology, Kevin St, Dublin 8, Ireland \\ ${ }^{3}$ Department of Neurology, St. Vincent's University Hospital, Dublin, Ireland \\ ${ }^{4}$ School of Medicine, University College Dublin, Dublin, Ireland \\ ${ }^{5}$ School of Medicine, Trinity College, Dublin, Ireland \\ E-mail: quinlivb@tcd.ie and reilly@tcd.ie
}

Received 28 September 2015, revised 28 June 2016

Accepted for publication 21 July 2016

Published DD MM 2016

\begin{abstract}
Objective. To date human kinematics research has relied on video processing, motion capture and magnetic search coil data acquisition techniques. However, the use of head mounted display virtual reality systems, as a novel research tool, could facilitate novel studies into human movement and movement disorders. These systems have the unique ability of presenting immersive 3D stimulus while also allowing participants to make ecologically valid movementbased responses. Approach. We employed one such system (Oculus Rift DK2) in this study to present visual stimulus and acquire head-turn data from a cohort of 40 healthy adults.

Participants were asked to complete head movements towards eccentrically located visual targets following valid and invalid cues. Such tasks are commonly employed for investigating the effects orientation of attention and are known as Posner cueing paradigms. Electrooculography was also recorded for a subset of 18 participants. Main results. A delay was observed in onset of head movement and saccade onset during invalid trials, both at the group and single participant level. We found that participants initiated head turns $57.4 \mathrm{~ms}$ earlier during valid trials. A strong relationship between saccade onset and head movement onset was also observed during valid trials. Significance. This work represents the first time that the Posner cueing effect has been observed in onset of head movement in humans. The results presented here highlight the role of head-mounted display systems as a novel and practical research tool for investigations of normal and abnormal movement patterns.
\end{abstract}

S Online supplementary data available from stacks.iop.org/JNE/0/000000/mmedia

Keywords: attention, HMD, head turn, Posner, movement, virtual reality

(Some figures may appear in colour only in the online journal)

\section{Introduction}

It is well documented that the appearance of novel visual targets outside our central field of view results in a deflection

${ }^{6}$ Author to whom any correspondence should beaddressed. of gaze towards the appearing stimulus [1]. Furthermore, it has been established that these shifts in gaze are generated through a combination of distinct head, and eye movements [2-4] for small displacements. The command to shift one's gaze in response to a salient stimulus is generated in the superior colliculus and separated downstream into these 
distinct head and eye commands [5, 6]. While saccades have been well characterised through electrooculography (EOG) and retinal eye tracking studies [7-9], the role of head movement in gaze displacement has been less widely investigated [10]. This may be due, in part, to the relative difficultly involved in recording head movements in an accurate and objective manner. Early research into head kinematics relied on subjective observational studies or objective studies that recorded induced voltage in a sensor coil inside two perpendicular horizontal magnetic fields [11-13]. The introduction of motion capture technology has found utility across a wide range of kinematics research, including investigations of the contribution of directed head and body movements into larger displacements in gaze in control $[14,15]$ and clinical $[16,17]$ cohorts. These studies employ eccentrically located LEDs as peripheral visual targets and recorded saccade activity with EOG for comparison with head and body movements during deflections in gaze. While these studies have produced robust and accurate objective measures of head movement, paradigms built around motion capture systems do come with a number of unavoidable drawbacks. Motion capture systems require large open spaces, lack portability, are prohibitively expensive and are limited by the choice of stimulus and mode of stimulus presentation. We posit that virtual reality head mounted displays (HMD) may represent an alternative to motion capture for such studies while also addressing many of the limitations of motion capture systems.

The Posner cueing paradigm [18] is a neurological test often employed to assess an individual's ability to perform covert attentional shifts under different experimental conditions. The task has been applied to investigate manual (button-press) [19-21] and automatic (saccade) [22-24] reaction times to target stimuli with the aim of investigating the effects of covert orienting of attention in response to different cue conditions. Variations of the Posner cueing task have been applied in many areas of research and have proven to be very useful in assessing the effects of focal damage or disorders of attention [25-27] as well as increasing the understanding of spatial attention in healthy populations [28, 29]. Here it is hypothesised that the Posner effect, which has been widely demonstrated in automatic saccade responses, would also be present in the ecologically valid onset of head movement, given the well-established link between head movement and saccade activity during displacement of gaze [3, 14, 30, 31].

The aim of this study was to highlight how newly emerging technologies can be employed to record head kinematics and answer important oculomotor research questions. Here we utilise the capabilities of a virtual reality HMD (Oculus Rift DK 2) to examine the effects of orientation of attention on onset of head movement in a Posner style paradigm. The HMD presents an immersive 3D task that is updated at a rate of $75 \mathrm{~Hz}$ to reflect the movements of the user. Here the device is employed to collect head turn trajectories in 3 axis (yaw, pitch and roll) at up to $\sim 1000 \mathrm{~Hz}$ for offline analysis. To allow for comparisons of head movements and saccade activity, simultaneous EOG was also collected for a subset of participants.

\section{Materials and methods}

\subsection{Participants/ethics}

Forty healthy adults (21 men) (mean age \pm SD: $26.7 \pm 2.9$ years) participated in this study. All participants had normal or corrected to normal vision. In accordance with the Declaration of Helsinki, all participants gave their written informed consent to the study, which was approved by the Faculty Ethics Committee of the Faculty of Health Sciences at Trinity College Dublin.

\subsection{The task}

The task employed in the current study is a modified Posner task [18]. All recordings were carried out in a quiet room; participants were seated in a ridged, upright chair. The straps of the HMD were adjusted for each participant to ensure the unit was secure and comfortable. Before each test, the HMD was calibrated to ensure correct alignment between the virtual world and the participant's chair. At the beginning of each trial, a small focal sphere appeared at eye level at the $0^{\circ}$ position (figure 1(a)). The sphere's colour was black when the yaw of the HDM was greater than $\pm 5^{\circ}$ and green when the yaw was less than $\pm 5^{\circ}$. To elicited a cue and begin a trial, participants were required to maintain a yaw of less than $\pm 5^{\circ}$ for $1 \mathrm{~s}$ continuously. The cue (left or right pointing arrow) then appeared in place of the green sphere for $0.2 \mathrm{~s}$. The cue was then removed and a larger target sphere appeared in one of six possible locations (eye level; $\pm 30^{\circ}, \pm 45^{\circ}$ or $\pm 60^{\circ}$ ) (figure 1(b)). All target spheres were equidistant from the participant at $6 \mathrm{~m}$ and had a diameter of $60 \mathrm{~cm}$. Target spheres were coloured red when the yaw of the HMD was outside $\pm 5^{\circ}$ of the target's location and changed to blue inside this range. To end a trial, participants were required to maintain a yaw within $\pm 5^{\circ}$ of the target for $1.5 \mathrm{~s}$ continuously. At this point, the target sphere was removed and replaced with the smaller focal sphere at the $0^{\circ}$ location, marking the end of a trial. Valid cues were shown in $80 \%$ of trials, invalid cues were shown in the remaining $20 \%$. Participants were asked to complete a total of six blocks; each block consisted of 20 trials (120 trials). Trials were presented in a pseudo-random order across all blocks such that 16 valid and four invalid trials were presented for each of the six target locations. Participants were also asked to complete a shorter 15 trial practise block to help eliminate unwanted learning effects during the initial blocks. Participants were instructed to complete each trial in the shortest possible time. Targets at $\pm 30^{\circ}$ and $\pm 45^{\circ}$ appeared within the field of vision of participants at a neutral $0^{\circ} \pm 5^{\circ}$ positions, targets at $\pm 60^{\circ}$ were not visible from this neutral position. A video demonstration has been included in the supplementary section, this video shows a participant completing a number of trials and also a screen capture taken from the HMD, a sample frame from this video is shown in figure 1(b). It is important to note that this task represents a deviation from the traditional Posner paradigm in that targets do not appear in central vision. 


\title{
a Experimental design: Stimuli presentation order
}

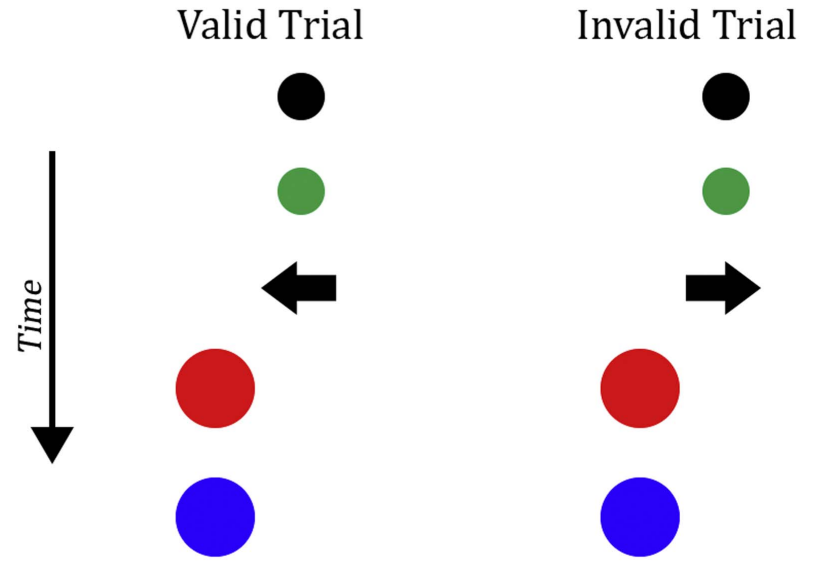

\author{
Focal Sphere $\left(\right.$ Yaw $\left.> \pm 5^{\circ}\right)$ \\ Focal Sphere $\left(\right.$ Yaw $\left.< \pm 5^{\circ}\right)$ \\ 1 second \\ Cue \\ 0.2 seconds \\ Target Sphere $\left(\right.$ Yaw $\left.> \pm 5^{\circ}\right)$ \\ Target Sphere $\left(\right.$ Yaw $\left.< \pm 5^{\circ}\right)$ \\ 1.5 seconds
}

\section{b Experimental design: Stimuli positions}
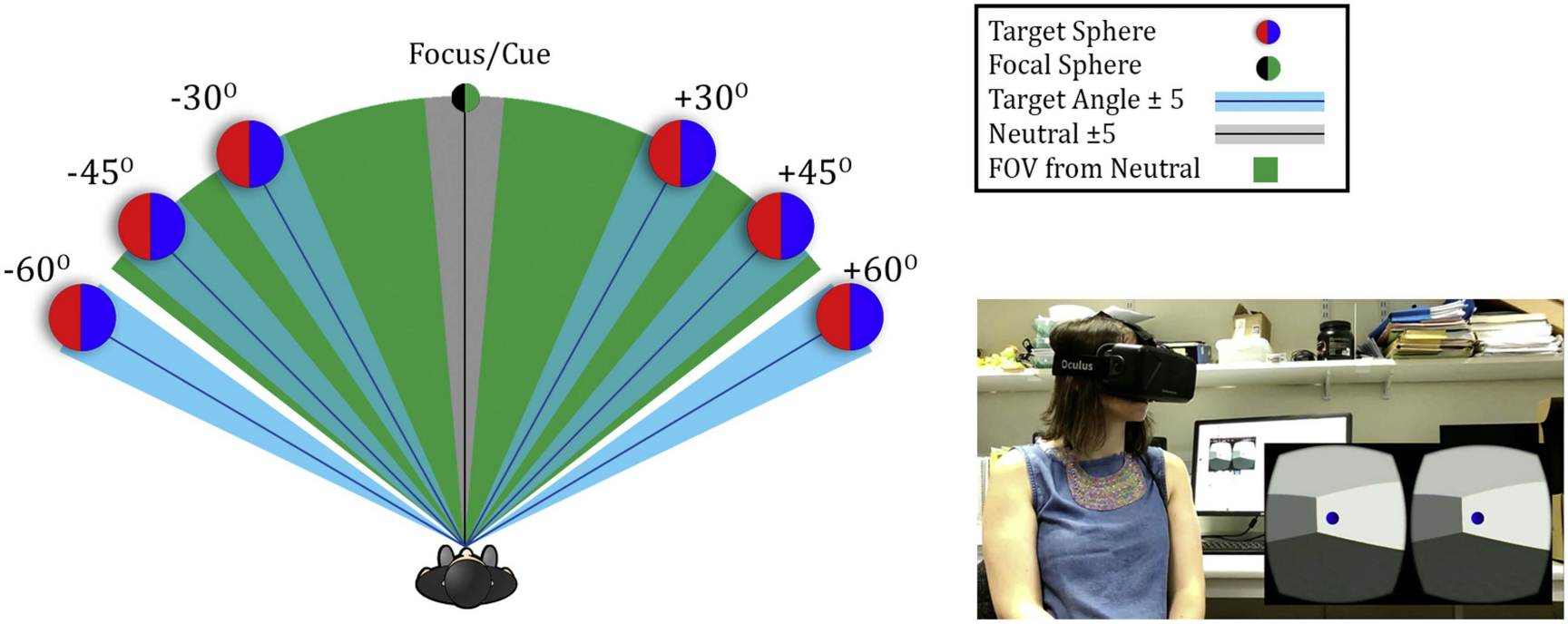

Figure 1. This figure shows an overview of the experimental design employed in the current study. Panel (a) contains an illustration of a valid and an invalid trial. This illustration depicts the order and timing of stimuli presentation during valid and invalid trials. Panel (b) contains a schematic plan view of the experimental architecture. The green segment represents a participant's approximate field of view (FOV) at the $0^{\circ}$ position. Target locations and target angle ranges are also indicated. All stimuli were presented in a realistic environment using the stereo-3D capability of the HMD. A sample frame from the supplementary video is also show, this video shows a participant completing a number of trials from one block. A screen capture from the HMD is also displayed.

\subsection{Data acquisition}

Yaw, pitch and roll of the HMD were recorded for all 40 participants at a sample rate of up to $1000 \mathrm{~Hz}$. In 18 of these participants, EOG was also recorded. EOG data, sampled at $512 \mathrm{~Hz}$, was collected from two active electrodes using a BioSemi high impedance recording system. Electrodes were placed inferiorly and laterally of the left eye. CMS and DRL electrodes were placed on the mastoids. Stimulus was generated and presented using custom software developed with Unity Pro 4.1 and the Oculus Rift software development kit for Unity. Triggers were generated at the beginning of each trial (cue onset) by the Unity software and were sent to the BioSemi system for synchronisation of EOG data via a custom micro-controller USB interface.

\subsection{Data analysis}

2.4.1. Head turn analysis. The sample rate of the raw HMD data was dictated by the frame rate of the custom software. Due to changes in processing demand, this frame rate was variable between 600 and $1000 \mathrm{~Hz}$. To account for this, data were first resampled to $500 \mathrm{~Hz}$ using a cubic spline data interpolation. Given the high signal to noise ratio of this data, no further preprocessing steps were required. The data were epoched for each trial from $0.4 \mathrm{~s}$ pre-cue to trial completion $(2.5-3.5 \mathrm{~s}$ post cue). For each trial, the following metrics were calculated; movement onset (ms), outbound movement duration (ms), max outbound velocity $\left({ }^{\circ} \mathrm{s}^{-1}\right)$, return movement duration (ms), max return velocity $\left({ }^{\circ} \mathrm{s}^{-1}\right)$ and turning point $(\mathrm{ms})$. All metrics were 


\section{a HMD displacement and velocity profile: Representative trial}
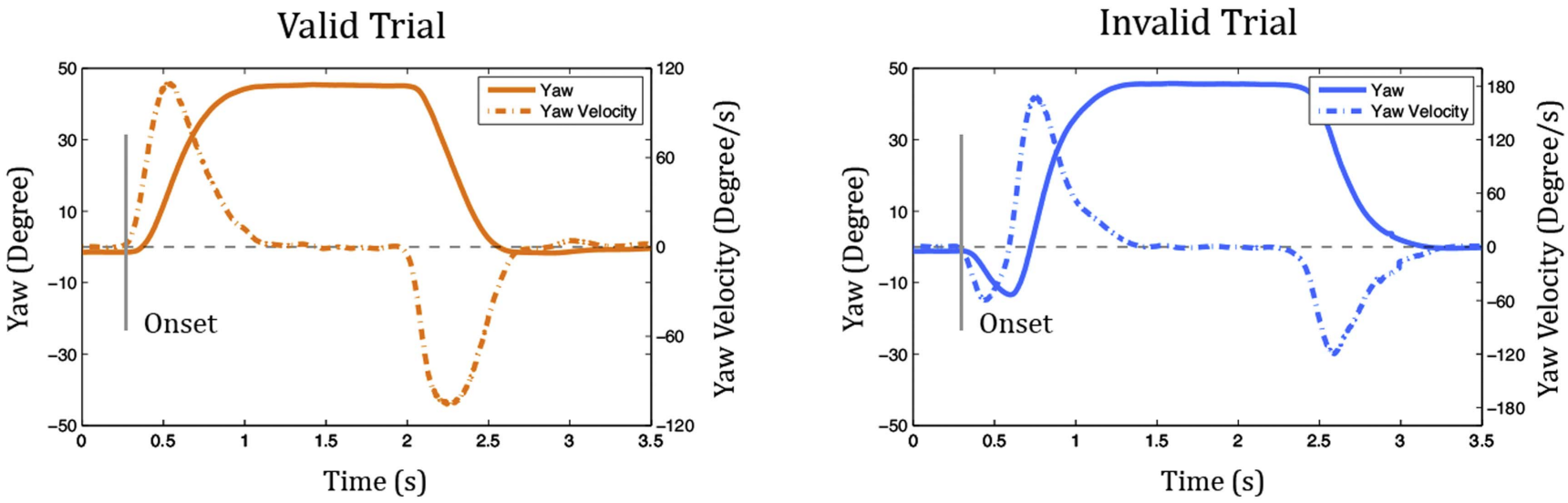

b HMD and EOG displacement profile: Representative trial
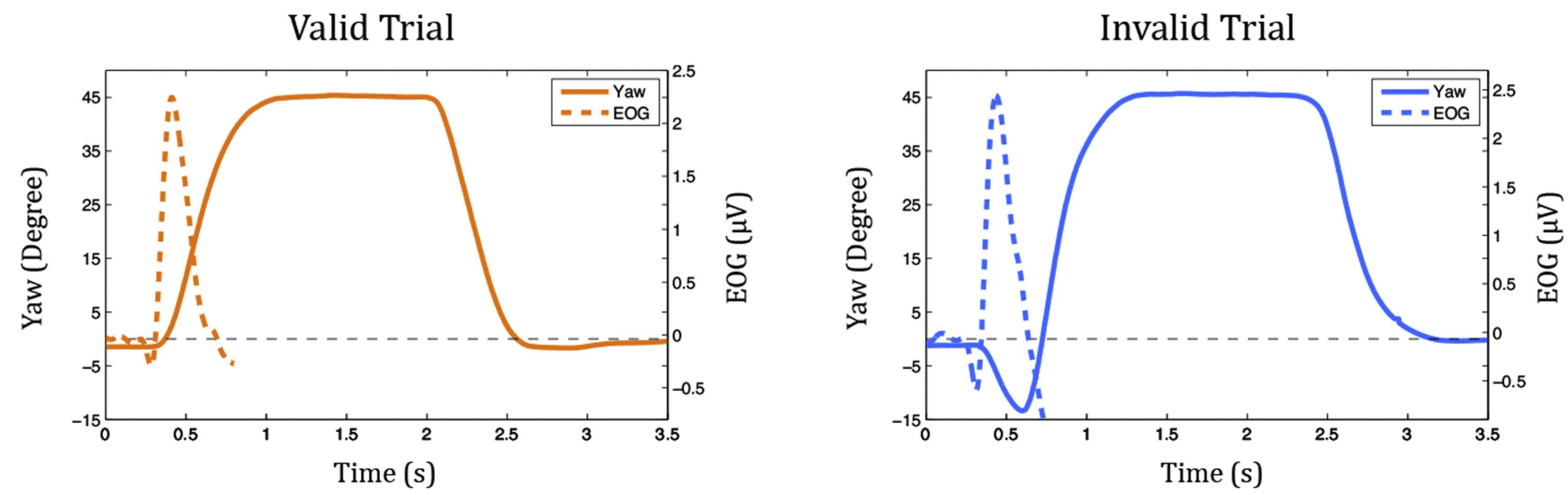

Figure 2. HMD and EOG data from a representative single valid and invalid $+45^{\circ}$ trial. Panel (a) contains yaw and yaw velocity profiles which are exploited for the movement parameter extraction algorithms described here. The solid line represents the resampled yaw data and the dot-dashed line represents the rate of change (velocity) of this data. The point of movement onset is represented as a vertical grey line. Panel (b) contains representative head yaw and EOG data from the same trial as panel (a). The solid line represents yaw data from the HMD sensors and is referenced to the left axis (degrees). The broken line represents EOG data and is referenced to the right axis $(\mu \mathrm{V})$.

calculated using yaw data. Both displacement and velocity were employed for detection the dependent metrics (figure 2). Velocity was calculated using the forward Euler differentiation method.

To find the point of movement onset, the maximum velocity and maximum displacement in the direction of the target were found. From the point of maximum velocity, the algorithm stepped back through the epoch until the velocity fell to $<20 \%$ of its maximum value and displacement fell to within $<30 \%$ of its maximum value continuously for 50 samples $(100 \mathrm{~ms})$. Onset of movement was defined as the latest of these samples. To find the outbound duration, the offset of the outbound movement was found and the time difference from onset to offset was calculated. Offset of outbound movement was defined at the first point the displacement reached $10 \%$ of its maximum displacement and its velocity dropped below $2^{\circ} \mathrm{s}^{-1}$. Max outbound velocity was defined as the maximum velocity during the outbound movement. To determine the return movement duration, the onset of return movement and offset of return movement were calculated. This onset was defined as the first time the displacement dropped $10 \%$ from its maximum and velocity exceeded its $2^{\circ} \mathrm{s}^{-1}$ threshold. The offset was defined as the first point the displacement dropped below $90 \%$ of its maximum and the velocity dropped below $2^{\circ} \mathrm{s}^{-1}$. The max return velocity was defined as the maximum velocity during the return movement. Turning point refers to invalid cues were participants initiate movement in the direction of the invalid cue and then need to change direction towards the actual location of the target. The turning point is defined as the point of max displacement in the invalid direction. This parameter is only informative in invalid cues and will return a value of $\approx 0$ on valid trials.

2.4.2. Saccade onset analysis. EOG data were analysed using custom MATLAB R2013b scripts and EEGLAB [32]. 
First, data were de-trended by removing the line of best fit from each channel to correct for signal drift. The data were then filtered using a 4th order band-pass Butterworth filter with a pass band of $0.1-10 \mathrm{~Hz}$. Next, EOG data from each trial were epoched with respect to cue onset, $1 \mathrm{~s}$ of data was used for each trial (399 ms pre- to $600 \mathrm{~ms}$ post-stimulus). Artefact rejection was carried out separately for each channel of EOG. If both channels were rejected, the trial was removed from further analysis. In all other cases, the mean of the remaining channels was employed for further analysis. Channels were marked as artefacts if they contained an absolute value of greater than $10 \mu \mathrm{V}$ or if they were uncorrelated with the other channel of EOG $(p>0.001)$.

Onset of EOG was detected for comparison with onset of head movement. The head movement outbound onset detection algorithm, as described above, was also employed for EOG data; letting the EOG data to correspond to displacement and its first derivative therefore correspond to velocity. Other parameters, as calculated for the HMD data, were not reliably obtainable using EOG, as such, onset of saccade was the only parameter extracted in this case.

\subsection{Statistical analysis: group level}

2.5.1. Analysis of variance (ANOVA). Each of the six behavioural measures extracted from the HMD data, as described in the previous section, were submitted to same ANOVA $(N=40)$. Onset of saccade, as extracted from EOG data, was also submitted to this ANOVA $(N=18)$. This analysis was designed to explore the effects of target angle, target side and cue validity on participant performance. Each parameter was submitted to a 3-way ANOVA. The independent variables used for this analysis were cue validity (level $=2$; valid and invalid), target side (level $=2$; left and right) and target angle (level $=3 ; 30^{\circ}, 45^{\circ}$ and $60^{\circ}$ ).

Head movement onsets and saccade onsets were also submitted to an ANOVA for the subset of participants for whom EOG was collected $(N=18)$. This analysis was carried out to probe the relationship between head and eye movement onset during eccentric deflections in gaze. A 4-way ANOVA was employed with the independent variables data type (level $=2, \quad$ HMD and EOG), cue validity (level $=2$; valid and invalid), target side (level $=2$; left and right) and target angle (level $=3 ; 30^{\circ}, 45^{\circ}$ and $60^{\circ}$ ).

2.5.2. Regression analysis. To further explore the relationship between head movement onset and saccade onsets, two linear regression models were created using valid and invalid trials. The dependant variable of these models was saccade onset and the predictor variable was head movement onset. Each model was created using onset data from all possible target locations. In cases where trials had been rejected from the EOG data due to artefact detection, the corresponding trials were also rejected from the HMD data for use in the regression model. In the valid model 1303 out of a possible $1728(75.41 \%)$ trials were maintained, in the invalid model 380 out of a possible 432 trials $(87.96 \%)$ were maintained.

\subsection{Statistical analysis: single participant level}

To test the utility of the current task as an investigative tool, some follow up single participant statistical analysis was carried out. The primary aim of this analysis was to show that the Posner effect, which was observed at group level, was reproducible at a single participant level, i.e. invalid cues should result in a slower onset of head movement when the target sphere is visible at the neutral position. In line with this definition, only targets from $\pm 30^{\circ}$ to $\pm 45^{\circ}$ were employed for this analysis, as targets at $\pm 60^{\circ}$ were not initially visible and therefore do not represent a Posner task per se. For each participant, all valid trials from $\pm 30^{\circ}$ to $\pm 45^{\circ}$ were grouped, as were all invalid trials for the same angles. These two groups were then submitted to a 2-sample unpaired t-test to determine if statistically significant differences existed due to trial validity, which would represent a Posner effect.

Next the regression analysis, as detailed above, was repeated at a single participant level to show that the relationship between head movement onset and saccade onset was also reproducible at the single participant level. Due to the limited amount of invalid data, this analysis was only carried out for valid trials, although results of the group level regression analysis suggest that this relationship should exist for both valid and invalid trials.

\section{Results}

\subsection{Head turn results}

3.1.1. Movement onset (Posner effect). The task employed in the current study represents a modified Posner cueing task. In this context, onset of head movement is employed rather than the typical button press or saccade response usually used for Posner tasks. The results presented here indicate that onset of head movement is delayed when the cue presented to participants is invalid (figure 3(a)). This delay in movement onset during invalid trials represents a Posner cueing effect; the 3-way ANOVA analysis revealed a highly significant effect of cue validity $(F(1,39)=144.9, p<0.001)$. There was also a significant main effect of target angle $(F(2$, $78)=3.2, p<0.05)$ suggesting that the position of the target in the participants field of view had some impact on movement onset. An interaction effect of cue validity and target angle was also observed $(F(2,78)=12.8, p<0.001)$ suggesting that the variation due to target angle was not consistent across valid and invalid trials. This is explained by the absence of a Posner effect at the $\pm 60^{\circ}$ target angles. As the target sphere is not initially visible, the contradiction between cue and target that cause the Posner effect is also absent. No other factors in this analysis were significant, a full summary of these results are displayed below (table 1).

3.1.2. Turning point. During invalid trials, when participants initiate movement in the invalid direction, a point of 


\section{Head movement parameters}
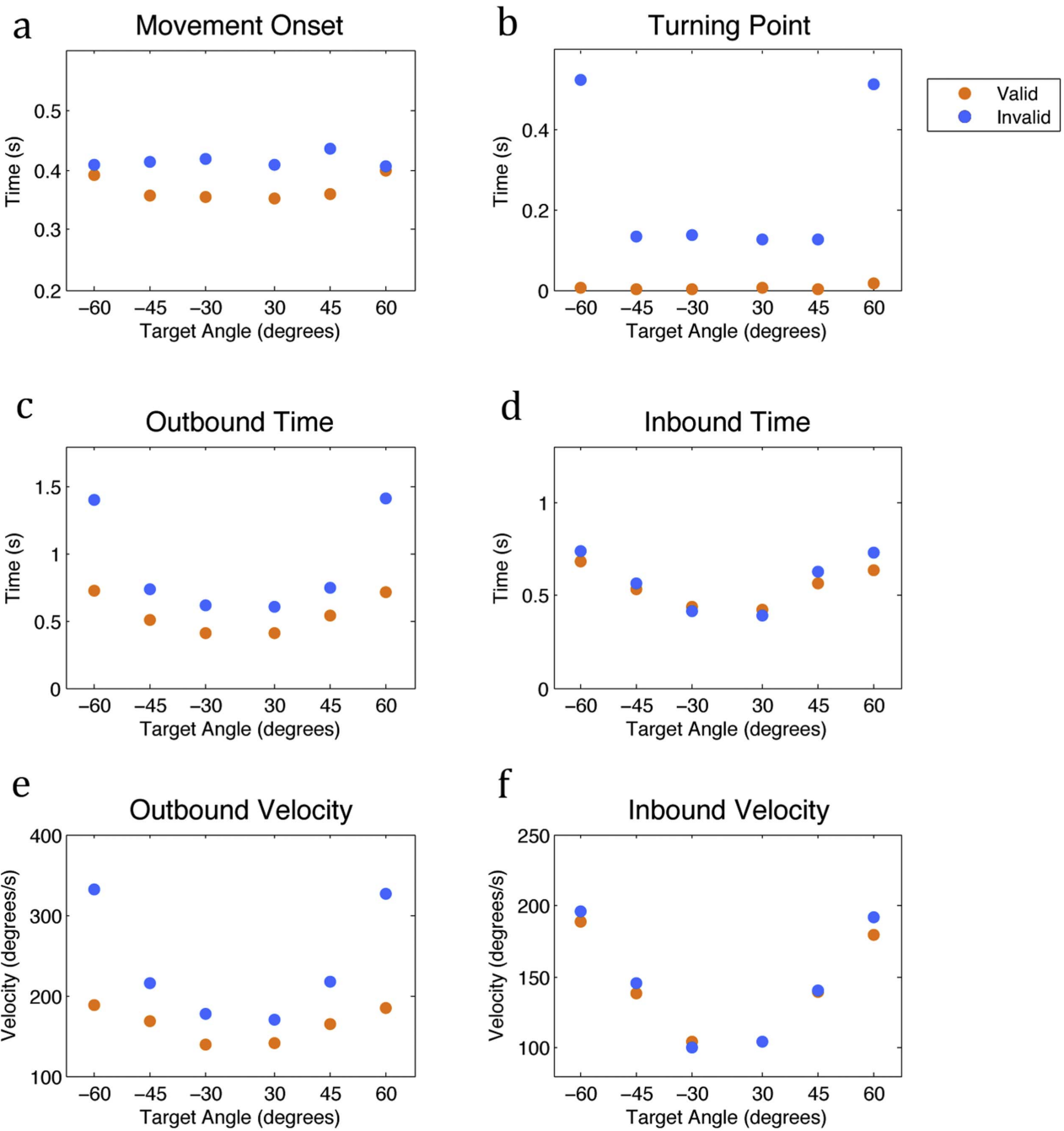

Figure 3. The six movement parameters as extracted from the head movement data. These plots represent group average data. All target angles ( $x$-axis) are displayed for each parameter as well as trial type (valid: orange, invalid: blue). Panel (a) shows movement onset times, panel (b) shows turning point times, panels (c) and (d) show outbound and inbound duration respectively and panels (e) and (f) show outbound and inbound maximum velocity respectively.

correction or a turning point is recorded. The results of the ANOVA analysis demonstrate a significant main effect of cue validity $(F(1,39)=219, p<0.001)$, a significant main effect of target angle $(F(2,78)=196.6, p<0.001)$ and a significant interaction effect of cue validly and target angle $(F(2,78)=150.2, p<0.001)$. Both the main effect of cue validity and the interaction effect of cue validity and target angle relate to the absence of a turning point in valid trials; a value of $\approx 0$ is returned in such cases. The significant effect of angle is more informative and is driven by the variance in invalid trials across target angles. Turning points for $\pm 30^{\circ}$ and $\pm 45^{\circ}$ are largely equivalent but turning points for $\pm 60^{\circ}$ tend to be significantly slower (figure 3(b)). This is because the target is visible from the start position during these $\pm 30^{\circ}$ and $\pm 45^{\circ}$ trials and so the participant will quickly realise the cue was invalid and correct their movement. During trials where the target is at $\pm 60^{\circ}$ the turning point is much later as participants need to partially complete an invalid movement to discover that the cue was invalid. This difference causes the significant effect of angle that has been observed here.

3.1.3. Outbound time. Outbound time is a measurement of the time taken for a participant to complete a movement towards the target location. Again, the results show a delay in the invalid trials. This delay corresponds to the delayed onset (Posner effect) and the fact the some movements are initiated in the invalid direction. Outbound time shows a statistically significant effect of cue validity $(F(1,39)=177.8$, $p<0.001)$, target angle $(F(2,78)=13.4, p<0.001)$ and an interaction effect of cue validity and target angle $(F(2$, $78)=92.6, p<0.001)$. No other effects were significant in outbound time (table 1 ).

3.1.4. Return time. Return time refers to the time taken for a participant to complete the return movement to the neutral 
Table 1. A summary of the 3-way repeated measure analysis of variance (ANOVA) results for the six movement parameters as depicted in figure 3. This table represents the group level data only. Degrees of freedom (df), $F$ - and $p$-values are reported for all tests. The 3 factors of interest in this analysis are cue validity (Valid), target side (Side) and target angle (Angle).

\begin{tabular}{|c|c|c|c|c|c|c|c|}
\hline \multirow[t]{2}{*}{ Onset } & \multicolumn{7}{|c|}{ Turning point } \\
\hline & df & $F$ & $p$ & & df & $F$ & $p$ \\
\hline Valid & $(1,39)$ & 144.9 & $<0.00$ & Valid & $(1,39)$ & 219 & $<0.0$ \\
\hline Side & $(1,39)$ & 0.9 & 0.33 & Side & $(1,39)$ & 0.1 & 0.78 \\
\hline Angle & $(2,78)$ & 3.2 & $<0.05$ & Angle & $(2,78)$ & 196.6 & $<0.001$ \\
\hline Valid*Side & $(1,39)$ & 0.1 & 0.78 & Valid*Side & $(1,39)$ & 1.1 & 0.32 \\
\hline Valid*Angle & $(2,78)$ & 12.8 & $<0.001$ & Valid*Angle & $(2,78)$ & 150.2 & $<0.001$ \\
\hline Side*Angle & $(2,78)$ & 2.1 & 0.13 & Side*Angle & $(2,78)$ & 0.1 & 0.92 \\
\hline Valid*Side*Angle & $(2,78)$ & 1.5 & 0.23 & Valid*Side*Angle & $(2,78)$ & 0.3 & 0.74 \\
\hline \multirow[t]{2}{*}{ Outbound time } & & & & Return time & & & \\
\hline & df & $F$ & $p$ & & df & $F$ & $p$ \\
\hline Valid & $(1,39)$ & 177.8 & $<0.001$ & Valid & $(1,39)$ & 4.1 & 0.06 \\
\hline Side & $(1,39)$ & 0.3 & 0.58 & Side & $(1,39)$ & 0.1 & 0.98 \\
\hline Angle & $(2,78)$ & 13.4 & $<0.001$ & Angle & $(2,78)$ & 67.3 & $<0.001$ \\
\hline Valid*Side & $(1,39)$ & 0.1 & 0.99 & Valid*Side & $(1,39)$ & 0.4 & 0.5 \\
\hline Valid*Angle & $(2,78)$ & 92.6 & $<0.001$ & Valid*Angle & $(2,78)$ & 2.1 & 0.12 \\
\hline Side*Angle & $(2,78)$ & 0.4 & 0.68 & Side*Angle & $(2,78)$ & 2.4 & 0.1 \\
\hline Valid*Side*Angle & $(2,78)$ & 0.4 & 0.65 & Valid*Side*Angle & $(2,78)$ & 0.3 & 0.74 \\
\hline \multirow[t]{2}{*}{ Outbound velocity } & & & & Return velocity & & & \\
\hline & df & $F$ & $p$ & & df & $F$ & $p$ \\
\hline Valid & $(1,39)$ & 80.4 & $<0.001$ & Valid & $(1,39)$ & 2.4 & 0.13 \\
\hline Side & $(1,39)$ & 0.3 & 0.58 & Side & $(1,39)$ & 1.1 & 0.31 \\
\hline Angle & $(2,78)$ & 170.8 & $<0.001$ & Angle & $(2,78)$ & 194.1 & $<0.001$ \\
\hline Valid ${ }^{*}$ Side & $(1,39)$ & 0.1 & 0.83 & Valid*Side & $(1,39)$ & 0.1 & 0.76 \\
\hline Valid*Angle & $(2,78)$ & 87.6 & $<0.001$ & Valid*Angle & $(2,78)$ & 3.1 & 0.067 \\
\hline Side*Angle & $(2,78)$ & 0.1 & 0.87 & Side*Angle & $(2,78)$ & 1.5 & 0.22 \\
\hline Valid*Side*Angle & $(2,78)$ & 0.6 & 0.55 & Valid*Side*Angle & $(2,78)$ & 0.8 & 0.46 \\
\hline
\end{tabular}

position after trial completion. As expected, the results show that larger movements take longer to complete. The results of the ANOVA analysis support this, showing a significant effect of target angle $(F(1,39)=67.3, p<0.001)$. No other main or interactions effects were significant (table 1).

3.1.5. Outbound velocity. Outbound velocity is a measure of the maximum velocity of yaw during the outbound movement. The results suggest that larger movements tend to result in larger peak amplitude, as might be expected. In the valid trials this can be seen as a linear increase with target angle. Invalid trials tend to have a larger peak velocity as subjects often move in the invalid direction initially before correcting back to the valid direction. This is most noticeable in the $\pm 60^{\circ}$ condition where the target is outside the field of view at cue presentation. In such cases the participant could be completing a movement of up to $120^{\circ}$, resulting in the larger peak velocity seen. The results of the 3-way ANOVA show a statistically significant effect of cue validity $(F(1$, $39)=80.4, p<0.001)$, target angle $(F(2,78)=170.8$, $p<0.001)$ and an interaction effect of cue validity and target angle $(F(2,78)=87.6, p<0.001)$. No other factors or interaction were statistically significant for outbound velocity (table 1$)$

3.1.6. Return velocity. Return velocity is a measure of the peak velocity of yaw during a post-trial return movement. The results show that larger movements tend to contain a larger peak velocity. This observation agrees with that of the outbound velocity results. The results of the 3-way ANOVA show a significant effect of target angle $(F(2,78)=194.1$, $p<0.001)$. No other main or interaction effects were significant for return velocity (table 1).

\subsection{Saccade onset analysis}

Saccade onset is often employed as an ecologically valid response for Posner style paradigms. To test the hypothesis that the proposed paradigm would elicit a delayed onset of saccade during invalid trials, all EOG onsets were submitted to a 3-way ANOVA. This analysis revealed a highly significant effect of cue validity $(F(1,17)=81.9, p<0.001)$, representing a confirmation of the Posner cueing effect in saccade onset. There was also a significant main effect of target angle $(F(2,34)=10.9, p<0.001)$ and a significant 
Table 2. A summary of the 3-way repeated measure analysis of variance (ANOVA) results for EOG onset data. This table represents the group level data. Degrees of freedom (df), $F$ - and $p$-values are reported for all tests. The 3 factors of interest in this analysis are cue validity (Valid), target side (Side) and target angle (Angle).

\begin{tabular}{llrc}
\hline EOG onset & & & \\
& df & \multicolumn{1}{c}{$F$} & $p$ \\
\hline 1. Valid & $(1,17)$ & 81.9 & $<0.001$ \\
1. Side & $(1,17)$ & 0.6 & 0.46 \\
1. Angle & $(2,34)$ & 10.9 & $<0.001$ \\
1. Valid*Side & $(1,17)$ & 0.5 & 0.49 \\
1. Valid*Angle & $(2,34)$ & 13.5 & $<0.001$ \\
1. Side*Angle & $(2,34)$ & 0.3 & 0.74 \\
1. Valid*Side*Angle & $(2,34)$ & 0.5 & 0.62 \\
\hline
\end{tabular}

interaction effect of cue validity and target angle ( $F(2$, $34)=13.5, p<0.001)$ which is consistent with the onset of head movement results reported above. No other factors were significant in this analysis (table 2).

Changes in gaze are generated through a combination of eye, head, trunk and lower limb movements [15, 33, 34]. Furthermore, it has been observed that onset of saccades precede head movements in a well-characterised fashion [31]. In order to show this property of gaze under the current paradigm, EOG was collected during testing for a subset of 18 participants. For both valid and invalid trials it was observed that saccade onset preceded head movement onset for all target locations in a uniform fashion (figure 4(a)). This was verified in the ANOVA analysis. A statistically significant effect existed for cue validity $(F(1,17=144.7, p<0.001))$, representing the Posner effect which was observed in each modality independently. There was also a statistically significant effect of recording modality $(F(1,17=32.5$, $p<0.001)$ ), confirming that onset of eye movement occurs earlier than onset of head movement. The effect of target angle $((F(2, \quad 34=11.9, \quad p<0.001)))$ and interaction between target angle and cue validity $(F(2,34=10.2$, $p<0.001)$ ) mirror the results that were observed during the individual 3-way analysis. The effect of target angle could suggest that onset is faster when targets are closer to the central field of vision but further research is required to draw a definitive conclusion on this point. Finally the interaction between cue validity and recording modality $(F(1,17=5.3$, $p<0.05)$ ) highlights that the Posner effect observed for saccade onsets is larger than that observed for onset of head movement, as may be observed from figure 4 . A full summary of this analysis is displayed in table 3 .

In order to further probe the relationship between head movement onset and saccade onset, a linear regression model was created (figure 4(b)). The aim of this model was to demonstrate the strength of the relationship. In the case of valid trials, a significant regression equation was found ( $F(1$, $1301)=761.8, p<0.001)$, with an adjusted $R^{2}$ value of
0.37. Participant's predicted onset of eye movement is equal to $0.067+0.626$ (HMD Onset) seconds. The intercept value was significant in this model $(t=7.3, p<0.001)$ as was the HMD coefficient $(t=27.6, p<0.001)$. The model of invalid trials did not produced a significant regression equation ( $F(1$, $378)=0.6, p=0.45)$. This is likely due to the fact that there is a more narrow range of values available for invalid trials, making it more difficult to observe a statistically significant relationship.

\subsection{Single participant analysis}

Single participant analysis was carried out to test the utility of the current protocol at a single participant level. The protocols and task described here may have applications across a wide range of movement disorders. In such cohorts, group level analysis is often less informative due to increased inter-participant variability and single participant analysis may therefore be more applicable. The aim of this analysis was to demonstrate that both the Posner effect and head movement/ saccade onset relationship were reproducible a single participant level. As the Posner effect was only observed for targets at $\pm 30^{\circ}$ and $\pm 45^{\circ}$, these angles were employed for the single participant analysis.

To test if the Posner effect was detectable at an individual level, trials from $\pm 30^{\circ}$ and $\pm 45^{\circ}$ were grouped for valid $(N=64)$ and invalid $(N=16)$ trials and then submitted to a 2-sample unpaired t-test for each of the 40 participants. A statistically significant difference $(p<0.05)$ was observed in 36 out of 40 participants (90\% of participants). This result suggests that the Posner effect observed at group level is also highly reproducible at the single participant level.

To test the reproducibility of the saccade onset head movement onset relationship, the regression analysis was repeated at the single participant level. The results of this analysis reveal that this relationship is highly significant in 14 out of 18 participants $(p<0.05)$. $R$-values were also very consistent across participants $(0.47 \pm 0.32$, mean \pm SD). This analysis further highlights the uniformity with which saccade onsets lead head movement onsets during eccentric changes in gaze.

\section{Discussion}

In recent years, advances in sensing and display technologies have made virtual reality systems viable, and companies like Oculus VR have helped to make this technology widely available and affordable. These systems have the capacity to present immersive 3D stimulus while also tracking and recording movements of the user, making them suitable for research of human kinematics. In the current study ecologically valid head and eye movements during a modified Posner cueing task were investigated using one such virtual reality system. The aim of this work was to show that these devices could collect robust data and have utility in the research of movement and movement disorders. 


\section{a HMD vs. EOG: Movement onset}
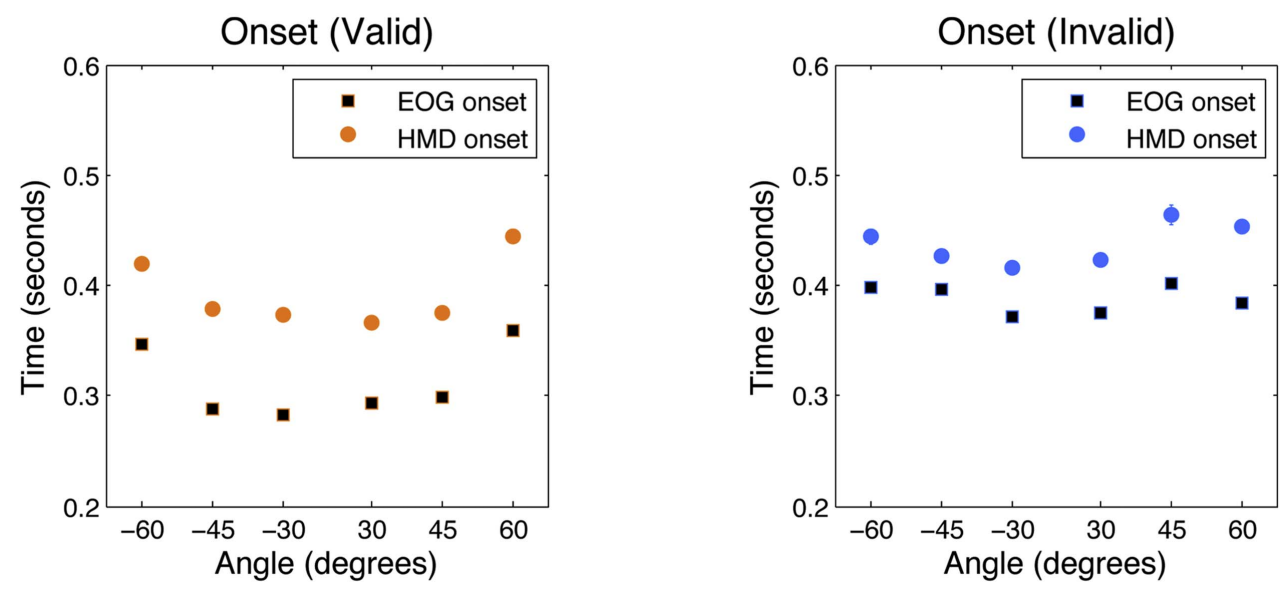

\section{b Regression analysis}
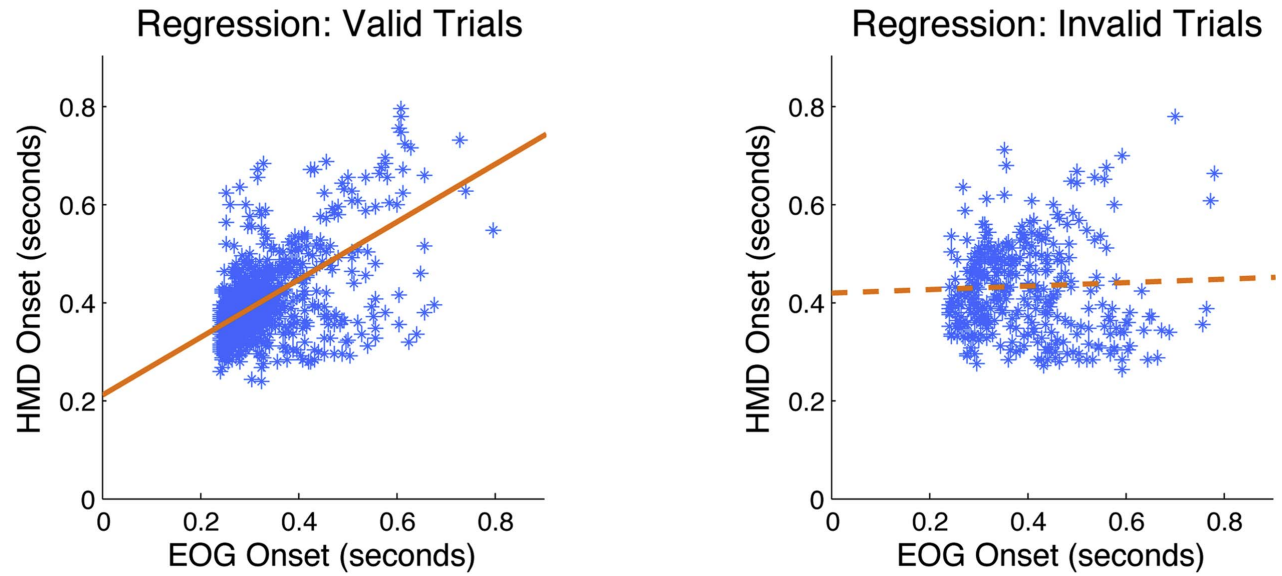

Figure 4. A plot of movement onsets for both head (solid) and eye (black centre) movements. The left plot represents valid trials (orange) and the right plot represents invalid trials (blue). These plots represent group mean data for all possible experimental conditions. Panel (b) contains a visualisation of the group level regression analysis. The left plot represents the model created employing all valid trials while the right plot represents the model created employing all invalid trials. The orange line represents the regression equation, which is highly significant in both cases.

Humans and other primates have evolved an elegant oculomotor system to better utilise images acquired by the retina. The oculomotor system is comprised of both low-level circuitry to rapidly reposition our line of site onto targets of interest, and high-level circuitry to implement flexible optimisation strategies. For example, dynamic orientation of visuospatial attention based on environmental cues enables us to react faster to salient stimulus appearing in our peripheral vision, as illustrated by the Posner cueing effect. The Posner spatial cueing effect [18] has been widely investigated using both manual button press [19-21] and automatic saccade [2224] reaction times to help investigate the effects of covert orienting of attention in response to different cue conditions. In the current study, a modified Posner task was employed and reaction times of both saccade onset and head movement onset were investigated. Although saccade onsets are commonly employed in this context, this work, to our knowledge, represents the first time that onset of head movement has been investigated as the response for such a task in humans. Onset of head movement has been used in a Posner style task for the investigation of attentional influence on sound-localisation behaviour in barn owls $[35,36]$ and an effect of orientation of attention was observed.

Given that saccades are known to precede head movements during changes in gaze in a stereotyped manner $[3,14,30,31]$, it was hypothesised that the Posner effect should be present in onset of head movement in addition to onset of saccade. This relationship between onset of head movement and onset of saccade has been investigated in monkeys using neck electromyography and eye tracking methods [37]. Given the considerable inertia of the head, it was unknown whether express saccades were accompanied by a parallel command to the head from the low level circuitry of the oculomotor system. Corneil et al demonstrated that visual target presentation elicits time-locked, lateralised recruitment of neck muscles for both express and non-express saccades.

The results presented here indicate that the Posner effect is present in the onset of head movement data at both group 
Table 3. A summary of the 4-way repeated measure analysis of variance (ANOVA) results for HMD versus EOG data depicted in figure 4. This table represents the group level data. Degrees of freedom (df), $F$ - and $p$-values are reported for all tests. The four factors of interest in this analysis are cue validity (Valid), recording modality (Modality), target side (Side) and target angle (Angle).

\begin{tabular}{|c|c|c|c|}
\hline HMD and EOG onset & $\mathrm{df}$ & $F$ & $p$ \\
\hline 1. Valid & $(1,17)$ & 144.7 & $<0.001$ \\
\hline 1. Modality & $(1,17)$ & 32.5 & $<0.001$ \\
\hline 1. Side & $(1,17)$ & 3.1 & 0.01 \\
\hline 1. Angle & $(2,34)$ & 11.9 & $<0.001$ \\
\hline 1. Valid*Modality & $(1,17)$ & 5.3 & $<0.05$ \\
\hline 1. Valid*Side & $(1,17)$ & 0.1 & 0.89 \\
\hline 1. Modality ${ }^{*}$ Side & $(1,17)$ & 0.2 & 0.63 \\
\hline 1. Valid ${ }^{*}$ Modality*Side & $(1,17)$ & 0.7 & 0.39 \\
\hline 1. Valid*Angle & $(2,34)$ & 10.2 & $<0.001$ \\
\hline 1. Modality ${ }^{*}$ Angle & $(2,34)$ & 0.1 & 0.95 \\
\hline 1. Valid ${ }^{*}$ Modality ${ }^{*}$ Angle & $(2,34)$ & 0.3 & 0.77 \\
\hline 1. Side ${ }^{*}$ Angle & $(2,34)$ & 0.4 & 0.67 \\
\hline 1. Valid ${ }^{*}$ Side $^{*}$ Angle & $(2,34)$ & 1.8 & 0.19 \\
\hline 1. Modality ${ }^{*}$ Side $^{*}$ Angle & $(2,34)$ & 1.2 & 0.33 \\
\hline 1. Valid ${ }^{*}$ Modality*Side*Angle & $(2,34)$ & 0.2 & 0.81 \\
\hline
\end{tabular}

level and at a single participant level (in $90 \%$ of participants). The effect was also observed in the EOG data for the subset of 18 participants, consistent with the existing literature $[22,23,38]$. The analysis showed that the relationship between onset of head movement and onset of saccade activity is highly stereotyped at group level for both valid and invalid trials and also at the single participant level for valid trials. The four-way ANOVA analysis confirm that Posner effect is larger for saccade onsets, however, no other interaction effects were observed in relation to recording modality, supporting the view that the head and eye movement onsets are highly related.

The presence of the Posner effect in saccade onset has been well documented in the literature [22-24], as has the relationship between saccade onset and head movement onset [3, 14, 30, 31]; furthermore, this relationship has been demonstrated in the current study. Given these points, it is likely that the delay observed in onset of head movement during invalid trials is a novel representation of the classic Posner effect. Thus head movement onset is a measure of the effect of covert orienting of attention and might be a useful alternative to button press responses and eye tracking methods.

While the subset of EOG recordings was useful in the current study, the results of the regression analysis presented here would suggest that little additional information is gained from the EOG data. This implies that future studies in control cohorts could rely on HMD data alone. It is possible that the relationship between head movement onset and saccade onset might be altered in some clinical settings, thus any novel research in such populations could benefit from collecting both eye and head movement data initially.

To further highlight the versatility of the HMD as a research tool, five additional movement parameters were extracted from the HMD data. The parameters investigated here included turning point, outbound and return movement durations and outbound and return maximum velocities. Each of these parameters was extracted from yaw data, although pitch, roll and positional data are also available from the system and could be informative for other tasks or populations. The results of this analysis were largely as expected; larger movements resulted in longer durations and higher maximum velocities, cue validity was a highly significant factor for outbound movements but was less so for selfinitiated return movements. The results presented here show quantitative and qualitative similarity to those found using more traditional non-portable motion capture recording techniques [14, 15]. On their own and for a healthy cohort, these parameters are not very informative, however, they could have utility for making comparisons between different clinical groups in future work. The same can be said about $60^{\circ}$ targets included in the current paradigm, although these are not useful for investigating a Posner style question, the data collected during these trials may have utility for other research questions and its inclusion in the experiment makes this paradigm a more versatile research tool. Such movement parameters are easily extracted from the HMD data and in conjunction with novel paradigms, could be employed to answer specific and important research questions. The suitability of such parameters for research of abnormal movement in Parkinson's disease and dystonia has been demonstrated in studies that use motion capture technology to record whole body rotations during valid trials $[16,17]$.

\section{Conclusion}

Advances in commercial industries often lead to novel and important research tools. Here we posit that the advent of HMD virtual reality systems could represent a cost effective and portable measurement solution for many branches of kinematic research. The results presented here demonstrate that the current protocol can be employed to investigate the effects of covert orienting of attention through an ecologically valid movement-based response in cases where eye tracking may not be suitable or available. The current set of results also demonstrate how other movement parameters can be extracted from the HMD to answer a much wider set of research questions. Similar tasks could be created to measure the response of patients with movement disorders to novel interventions. It is also possible that the device could be employed as an intervention in itself, by creating novel training or rehabilitation tasks that could be beneficial for 
patients. In this work, it has been demonstrated, for the first time, that the Posner cueing effect is present in onset of head movement as well as onset of saccade activity in a group of healthy adult humans. This was possible due to the unique ability of the HMD to simultaneously present a task while using the movements of the user as an ecologically valid input in real time.

\section{Acknowledgments}

This work was primarily supported by a grant from the Health Research Board of Ireland (CSA 2012-5). The authors would like to thank the participants who donated their time as well as Niamh Foley, Siobhan O'Regan and Amy Restan for their help with data acquisition. The authors declare no competing financial interests that would bias the results reported here.

\section{Q1 References}

[1] Collewijn H 1977 Eye- and head movements in freely moving rabbits J. Physiol. 266 471-98

[2] Volle M and Guitton D 1993 Human gaze shifts in which head and eyes are not initially aligned Exp. Brain Res. 94 463-70

[3] Bizzi E, Kalil R E and Tagliasco V 1971 Eye-head coordination in monkeys: evidence for centrally patterned organization Science 173 452-4

[4] Chen L L, Lee D, Fukushima K and Fukushima J 2012 Submovement composition of head movement PloS One 7 e47565

[5] Freedman E G and Sparks D L 1997 Activity of cells in the deeper layers of the superior colliculus of the rhesus monkey: evidence for a gaze displacement command J. Neurophysiol. 78 1669-90

[6] Corneil B D and Munoz D P 2014 Overt responses during covert orienting Neuron 82 1230-43

[7] Huang Y F, Tan E G, Soon C S and Hsieh P J 2014 Unconscious cues bias first saccades in a free-saccade task Consciousness Cognition 29 48-55

[8] Watanabe M, Matsuo Y, Zha L, MacAskill M R and Kobayashi Y 2014 Fixational saccades alter the gap effect Eur. J. Neurosci. 39 2098-106

[9] Hollingworth A, Matsukura M and Luck S J 2013 Visual working memory modulates low-level saccade target selection: evidence from rapidly generated saccades in the global effect paradigm J. Vis. 134

[10] Shaikh A G, Wong A L, Zee D S and Jinnah H A 2013 Keeping your head on target J. Neurosci.: Official J. Soc. Neurosci. 33 11281-95

[11] Shaikh A G, Wong A, Zee D S and Jinnah H A 2015 Why are voluntary head movements in cervical dystonia slow? Parkinsonism Relat. Disorders 21 561-6

[12] Bremen P et al 2010 Applying double-magnetic induction to measure head-unrestrained gaze shifts: calibration and validation in monkey Biol. Cybern. 103 415-32

[13] Friston K J, Stephan K M and Frackowiak R S 1997 Transient phase-locking and dynamic correlations: are they the same thing? Hum. Brain Mapp. 5 48-57

[14] Hollands M A, Ziavra N V and Bronstein A M 2004 A new paradigm to investigate the roles of head and eye movements in the coordination of whole-body movements Exp. Brain Res. 154 261-6
[15] Anastasopoulos D, Ziavra N, Hollands M and Bronstein A 2009 Gaze displacement and inter-segmental coordination during large whole body voluntary rotations Exp. Brain Res. $193323-36$

[16] Anastasopoulos D, Ziavra N, Savvidou E, Bain P and Bronstein A M 2011 Altered eye-to-foot coordination in standing parkinsonian patients during large gaze and wholebody reorientations Mov. Disorders: Official J. Mov. Disorder Soc. 26 2201-11

[17] Anastasopoulos D, Ziavra N, Pearce R and Bronstein A M 2013 Trunk bradykinesia and foveation delays during whole-body turns in spasmodic torticollis J. Neurol. 260 2057-65

[18] Posner M I 1980 Orienting of attention Q. J. Exp. Psychol. 32 $3-25$

[19] Feldmann-Wustefeld T and Schubo A 2013 Textures shape the attentional focus: evidence from exogenous and endogenous cueing Attention Perception Psychophys. $\mathbf{7 5}$ 1644-66

[20] Rieth C A and Huber D E 2013 Implicit learning of spatiotemporal contingencies in spatial cueing J. Exp. Psychol. Hum. Perception Perform. 39 1165-80

[21] Johnson K N, Conture E G and Walden T A 2012 Efficacy of attention regulation in preschool-age children who stutter: a preliminary investigation J. Commun. Disorders $\mathbf{4 5}$ 263-78

[22] Dawel A, Palermo R, O'Kearney R, Irons J and McKone E 2015 Fearful faces drive gaze-cueing and threat bias effects in children on the lookout for danger Developmental Sci. 18 219-31

[23] Yokoyama T, Noguchi Y and Kita S 2012 Attentional shifts by gaze direction in voluntary orienting: evidence from a microsaccade study Exp. Brain Res. 223 291-300

[24] Chaminade T and Okka M M 2013 Comparing the effect of humanoid and human face for the spatial orientation of attention Frontiers Neurorobot. 712

[25] Ethier A A, Muckle G, Jacobson S W, Ayotte P, Jacobson J L and Saint-Amour D 2015 Assessing new dimensions of attentional functions in children prenatally exposed to environmental contaminants using an adapted Posner paradigm Neurotoxicol. Teratol. 51 27-34

[26] Reuter B et al 2011 Volitional saccades and attentional mechanisms in schizophrenia patients and healthy control subjects Psychophysiology 48 1333-9

[27] Lopez S G et al 2011 Attentional network task in schizophrenic patients and theirs unaffected first degree relatives: a potential endofenotype Actas Espanolas De Psiquiatria 39 32-44

[28] Wang C H, Liang W K, Tseng P, Muggleton N G, Juan C H and Tsai C L 2015 The relationship between aerobic fitness and neural oscillations during visuo-spatial attention in young adults Exp. Brain Res. 233 1069-78

[29] Fan J et al 2009 Testing the behavioral interaction and integration of attentional networks Brain Cognition 70 209-20

[30] Daye P M and Roberts D C 2015 Vestibulo-ocular reflex suppression during head-fixed saccades reveals gaze feedback control 35 1192-8

[31] Scharli A M, van de Langenberg R, Murer K and Muller R M 2013 Postural control and head stability during natural gaze behaviour in 6- to 12-year-old children Exp. Brain Res. 227 523-34

[32] Delorme A and Makeig S 2004 EEGLAB: an open source toolbox for analysis of single-trial EEG dynamics including independent component analysis J. Neurosci. Methods 134 9-21 
[33] Sklavos S, Anastasopoulos D and Bronstein A 2010 Kinematic redundancy and variance of eye, head and trunk displacements during large horizontal gaze reorientations in standing humans Exp. Brain Res. 202 879-90

[34] Bonnet C T, Morio C, Szaffarczyk S and Rougier P R 2014 Postural mechanisms to control body displacements in the performance of lateral gaze shifts J. Mot. Behav. 46 397-405

[35] Johnen A, Wagner H and Gaese B H 2001 Spatial attention modulates sound localization in barn owls J. Neurophysiol. 85 1009-12
[36] Kettler L and Wagner H 2014 Influence of double stimulation on sound-localization behavior in barn owls J. Comparative Physiol. A 200 1033-44

[37] Corneil B D, Olivier E and Munoz D P 2004 Visual responses on neck muscles reveal selective gating that prevents express saccades Neuron 42 831-41

[38] Langley L K, Friesen C K, Saville A L and Ciernia A T 2011 Timing of reflexive visuospatial orienting in young, youngold, and old-old adults Attention Perception Psychophys. 73 1546-61 


\section{QUERY FORM}

JOURNAL: Journal of Neural Engineering

Author: B Quinlivan et al

TITLE: Application of virtual reality head mounted display for investigation of movement: a novel effect of orientation of attention

ArTiCle ID: jneaa3518

The layout of this article has not yet been finalized. Therefore this proof may contain columns that are not fully balanced/ matched or overlapping text in inline equations; these issues will be resolved once the final corrections have been incorporated.

\section{SQ1}

Please be aware that the colour figures in this article will only appear in colour in the online version. If you require colour in the printed journal and have not previously arranged it, please contact the Production Editor now.

We have been provided funding information for this article as below. Please confirm whether this information is correct. Health Research Board: CSA 2012-5.

\section{Page 11}

Q1

Please check the details for any journal references that do not have a link as they may contain some incorrect information.

Page 11

Q2

Please provide the journal title in reference [30]. 